\title{
LAS FUENTES DE LA CONFIANZA SOCIAL*
}

\author{
FRANCISCO HERREROS VÁZQUEZ \\ Fundación Juan March. Madrid
}

\author{
PALABRAS CLAVE ADICIONALES \\ ADDITIONAL KEYWORDS \\ Confianza social, Capital social, Expectativas, Social Trust, Social Capital, Expectations, \\ Informacion. \\ Information.
}

RESUMEN. Uno de los conceptos clave en el análisis del capital social es la confianza social. La confianza social o confianza generalizada se define como confianza en desconocidos. A pesar de su centralidad para el análisis del capital social, los mecanismos que relacionan la pertenencia a redes sociales con el desarrollo de confianza social están generalmente ausentes. En este artículo se presentan dos vías a través de las que tienen lugar este tipo de mecanismos, a saber: como consecuencia de la participación en asociaciones y, de forma directa, a través del envío de señales que revisan las creencias iniciales acerca de lo dignos de confianza que son los desconocidos.

ABSTRACT. Social trust is one of the key concepts in the analysis of social capital. Social or generalized trust is defined as trust in unknown people. In spite of its centrality for the analysis of social capital, the mechanisms that connect membership to social network with the development of social trust are generally absent in the literature. This paper discusses two ways in which these mechanisms come about: (a) as a consequences of the participation in associations, (b) by sending signals that revise the initial beliefs about the trustworthiness of unknown people.

*Agradezco los comentarios de José María Maravall, Henar Criado y Margaret Levi.

E-mail: herreros@ceacs.march.es

Revista Internacional de Sociología (RIS)

Tercera Época, nº 35, Mayo-Agosto, 2003, pp. 151-175. 
RIS

BEVISTA INTERNACIONAL DE SOCIOLOGIA

No 35, Mayo-Agosto, 2003

FRANCISCO HERREROS VÁZQUEZZ

\section{INTRODUCCIÓN}

La literatura acerca del capital social, especialmente la realizada desde la ciencia política, concede una gran importancia a la confianza generalizada o confianza social. Para algunos autores, la noción de confianza social es la gran contribución de la literatura de capital social al programa investigador de la cultura política (Stolle, 2000). El concepto de confianza social parece importante para aquéllos que proclaman las bondades del capital social, debido a que gran parte de las externalidades positivas asociadas a la presencia del capital social se remiten, en mayor o menor medida, al capital social en forma de confianza generalizada. Las asociaciones voluntarias de la Italia cívica de Putnam son tan beneficiosas debido a que generan confianza social y permiten que personas desconocidas confien unas en otras. Las madres pueden dejar que sus hijos jueguen solos en los parques de Jerusalén porque, a diferencia de en Detroit, consideran que sus vecinos, por muy desconocidos que sean, son dignos de confianza (Coleman, 1990: 303)

La confianza social es para muchos autores un "juicio moral" que lleva a los individuos a pensar que la mayor parte de la población es digna de confianza (Uslaner, 1999: 124) Este "juicio moral" podría tener su origen, según los autores que siguen el enfoque culturalista hacia el capital social, en normas fruto de la socialización (Jackman y Miller, 1996: 635) Normalmente estas definiciones de la confianza social no van más allá: no se explica en qué sentido la confianza en desconocidos es un juicio moral, y tampoco se aclara cuáles son los procesos de socialización a través de los cuales los individuos pasan a desarrollar estas expectativas. Parte de estos problemas de análisis de la confianza social son atribuibles a la vinculación del concepto de confianza social al programa de investigación de cultura política. De hecho, los estudios de cultura política hace tiempo que han incluido en su catálogo de "actitudes" la confianza social. Inglehart (1990) incluye tres indicadores en su índice de cultura política de las democracias: confianza generalizada, satisfacción con su situación vital, y apoyo a un cambio revolucionario. Recientemente, Weingast (1997) también ha incluido la confianza dentro de los elementos culturales que dan estabilidad a la democracia. Ninguno de estos estudios parece haber superado las deficiencias teóricas que arrastra la tradición de cultura política, referidas en gran medida a problemas de dirección de la causalidad. Frente a los análisis más centrados en la idea de confianza como un "recurso cultural" (Sztompka, 1999: 14), en este trabajo se abordarán los problemas en torno a la confianza generalizada desde una perspectiva de elección racional.

La confianza generalizada es un tipo de confianza muy sensible a los comportamientos oportunistas. La confianza social o confianza generalizada es confianza en extraños, en desconocidos acerca de los que se carece de información acerca de si son o no dignos de confianza. Supone una creencia del tipo "creo que la gente en general es digna de confianza", o bien "creo que las personas, en general (con 
independencia de si van a volver a verme o no en el futuro) tienen un interés en no engañarme, en portarse conmigo de forma honesta". Alguien que tenga esas creencias peculiares acerca de las preferencias de los demás será muy fácil de engañar: tenderá a confiar en todo el mundo. Por el contrario, la confianza particularizada está basada en experiencias pasadas con otros individuos (Hardin, 1993), o bien en una evaluación de qué comportamiento es racionalmente esperable por parte del otro dados los incentivos presentes. Los comportamientos oportunistas son más evitables en este caso simplemente porque el individuo se expone menos a ellos. El misterio de la confianza social es que, si no se tiene información acerca del otro jugador, no hay base para saber si es o no digno de confianza. En ese caso, ¿por qué optar por confiar? Desde luego, si los dos jugadores son oportunistas, no hay ninguna razón para que confien. En este caso, el juego adoptaría la forma del "juego de la confianza" de Kreps (1990), que no es otra cosa que una versión del clásico dilema del prisionero de una sola ronda. La figura 1 es un diagrama de ese juego en forma extensiva.

Figura 1.

El juego de la confianza (Kreps, 1990: 100)

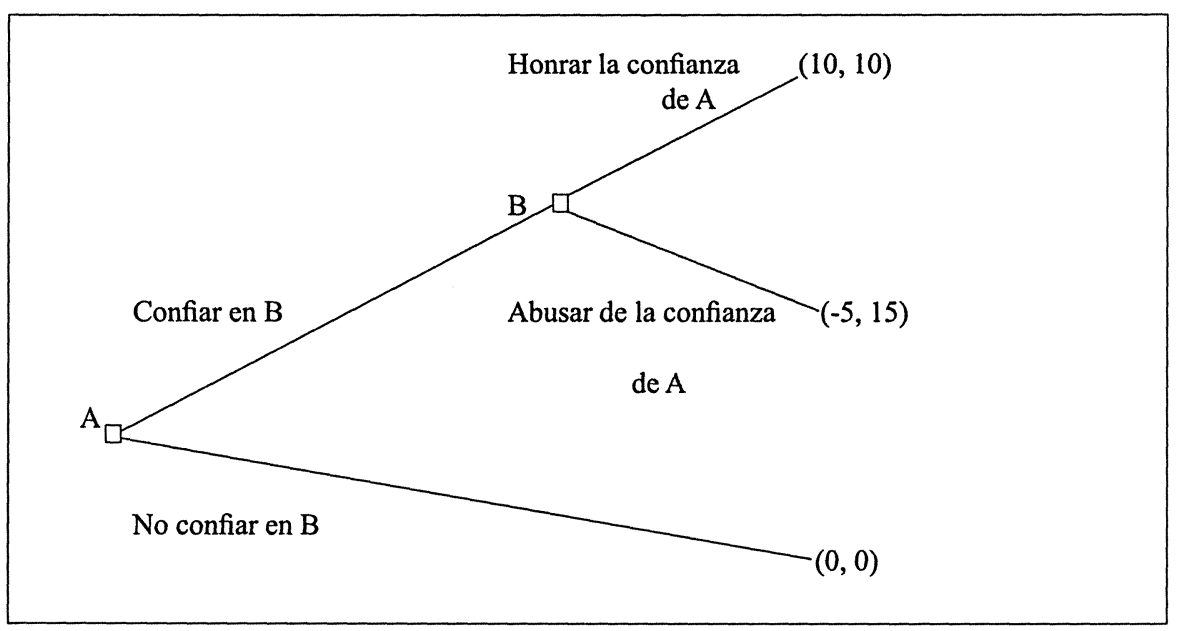

El resultado del juego de la confianza es que A decidirá no confiar en B. Y ello a pesar de que, de hecho, el juego de la confianza de Kreps es benévolo al menos con uno de los dos jugadores. Nótese en la figura 1 que, aunque $B$ es por desgracia un oportunista, la estructura de pagos de $\mathrm{A}$ es la de un tipo mucho más simpático: su primera preferencia es confiar en B y que este haga honor a la confianza que 
RIS

REVISTA INTERNACIONAL DE SOCIOLOGIA

№ 35, Mayo-Agosto, 2003

se le ha depositado. El juego de la confianza de Kreps es una versión unilateral de dilema del prisionero, en el sentido de que los jugadores tienen unos pagos asimétricos. Para entender mejor la lógica de este juego, podemos suponer que el jugador $\mathrm{A}$ es un comprador de coches usados que espera que el coche que le vende "el honrado B" no sea una chatarra. De ahí que su primera preferencia sea confiar en $\mathrm{B}$ y que $\mathrm{B}$ haga honor a esa confianza. La situación es algo distinta si se respeta el supuesto tradicional del dilema del prisionero de que ambos jugadores son oportunistas. La forma extensiva del juego de la confianza como un dilema del prisionero tradicional se recoge en la figura 2. El juego de la confianza es en este caso algo distinto. Ya no se trata, como en el caso de la figura 1, de un juego donde un individuo deposita su confianza en otro y este debe decidir si honrar esa confianza o coger el dinero y correr, sino más bien de un sistema de confianza en el que ambos confian y son depositarios de confianza. En este caso, ambos jugadores son oportunistas, es decir, ambos prefieren abusar de la confianza del otro. El resultado es que ninguno de los dos confía.

Tanto en el juego de la confianza de Kreps como en la versión más convencional de dilema del prisionero, este resultado puede ser evitado si jugamos el juego

Figura 2.

El juego de la confianza como un dilema del prisionero clásico

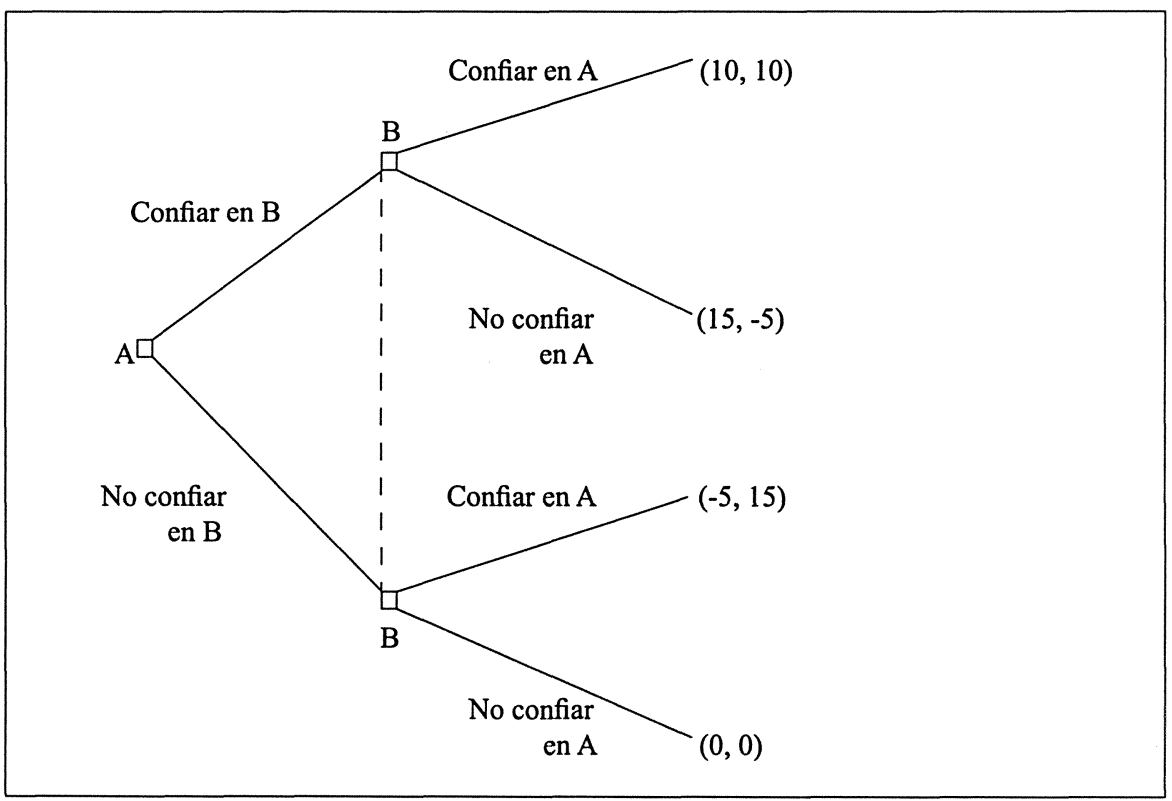


repetidas veces. En el caso de que el juego de la confianza se repita un número infinito de veces, o, lo que es más realista, ni A ni B sepan cuando se producirá una ronda final, entonces puede ocurrir que $\mathrm{A}$ decida confiar en $\mathrm{B}$, y que $\mathrm{B}$ a su vez confie en A. Una estrategia especialmente prometedora para alcanzar un equilibrio cooperativo en el juego de la confianza repetido es tit-for-tat: "Confiar en la primera ronda, y hacer luego lo que haya hecho el otro jugador en la ronda anterior" (Axelrod, 1984). Si ambos jugadores escogen tit-for-tat, el resultado del juego es que A y B confian, en el caso del juego de la confianza como dilema del prisionero convencional, o que $\mathrm{B}$ hace honor a la confianza que $\mathrm{A}$ ha depositado en él, en el caso del juego de la confianza de Kreps.

Por supuesto, un resultado en el que tit-for-tat fuese un equilibrio es sólo uno de los muchos resultados posibles del superjuego de dilema del prisionero. Un problema adicional de esta solución al dilema del prisionero es que su aplicación al mundo real es muy limitada: en términos generales, la cooperación es difícil de mantener cuando el juego no es repetido (o hay un juego final), cuando no hay información completa sobre los otros jugadores, cuando hay un gran número de jugadores, o cuando los jugadores no valoran suficientemente el futuro. En el

Figura 3.

El juego de la confianza social

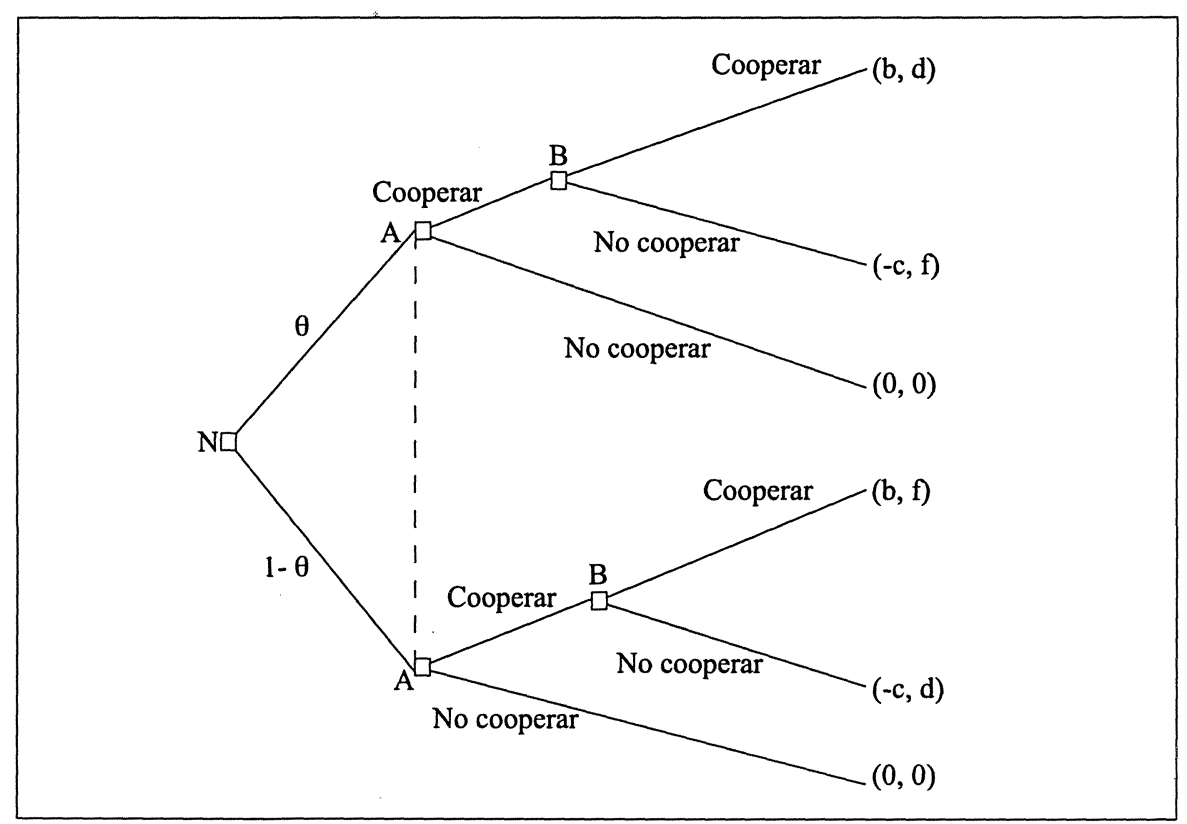


RIS

REVISTA INTERNACIONAL DE SOCIOLOCIÁ

№ 35, Mayo-Agosto, 2003

FRANCISCO HERREROS VÁZQUEZ

caso de interacciones impersonales, estas condiciones raramente se dan (North, 1990: 12-13; Elster, 1985: 360-361). En todo caso, si efectivamente se alcanza un resultado cooperativo, que ambos confien en el otro y honren la confianza que en ellos se ha depositado, este resultado estaría más próximo a la confianza particularizada que a la noción de confianza social. A través de la repetición del juego, cada uno aprende qué puede esperar del otro, y si vale la pena seguir con una estrategia tit-for-tat o cambiar a una estrategia en la que cada vez que el otro deja de honrar la confianza en él depositada, el primer jugador opta por no confiar en el segundo nunca más.

En el caso de la confianza social, la situación es algo distinta. La confianza social es confianza en desconocidos. En este caso, resulta interesante fijarnos en un juego de una sola ronda, más que en un juego repetido. Si el juego se repite nos internamos en otro tipo de juego de la confianza, referido, como ya hê dicho, a la confianza particularizada.

Nótese que el juego de Kreps es un juego de información perfecta, cierta y completa. Podemos solucionarlo fácilmente mediante inducción retrospectiva. Por otro lado, los movimientos del jugador $A$ confiar o no confiar, son idénticos a cooperar o no cooperar. Esto supone que el juego no recoge la idea de la confianza como una expectativa acerca de las preferencias de los demás. El juego de la confianza social es más bien el que se recoge en la figura 3. En este juego, los movimientos de los jugadores son distintos. "Confiar" y "hacer honor a la confianza" han sido sustituidos por "cooperar", y "desconfiar" y "no hacer honor a la confianza" han sido sustituidos por "no cooperar". Otra innovación son las probabilidades $\theta$ y $1-\theta$. Estas probabilidades se refieren a las expectativas del jugador A sobre lo digno de confianza que es el jugador $\mathrm{B}$. El jugador A tiene las mismas preferencias que en el juego de Kreps de la figura 1. No se trata de un oportunista: su primera preferencia es cooperar con el jugador B si B es digno de confianza. Este resultado tiene unos pagos de $b$ para el jugador A. Su segunda preferencia es no cooperar con $\mathrm{B}$, una acción que le deja tal cual estaba. Su última preferencia es cooperar con B y que este le engañe. En este caso, A incurriría en unos costes $\mathrm{c}$ (que en el árbol de decisión se recogen de forma negativa). El orden de sus pagos es, por tanto, $b>0>-c$. La mayor diferencia con el juego de la confianza de Kreps es que, en el juego de la figura 3, B puede ser de dos tipos. En las ramas superiores del árbol, B es una persona en la que se puede confiar. En las ramas inferiores nos encontramos con un jugador $\mathrm{B}$ decididamente poco recomendable. La diferencia entre ambos tipos de B se refleja en sus pagos. Un $\mathrm{B}$ digno de confianza obtendrá sus máximos pagos $(d)$ cuando decide honrar la iniciativa cooperativa de A. Sin embargo, si B es un oportunista, obtiene la utilidad más alta de engañar al jugador $A$. La segunda preferencia para un jugador $B$ digno de confianza es que $A$ decida no cooperar con él. Preferirá eso a engañar al jugador A. El orden de sus pagos es, por tanto: $d>0>f$. Un B oportunista, por su parte, tendrá como segunda preferencia que A no confia en él. Una segunda preferencia, 
por tanto, similar al jugador B digno de confianza, pero por razones distintas. El $\mathrm{B}$ oportunista preferirá no tener que establecer una relación de cooperación con $\mathrm{A}$, con los costes que eso le puede acarrear, por lo que su última preferencia será cooperar con A. El orden de sus pagos es, en consecuencia, $d>f>0$. El jugador $A$ debe decidir a qué tipo de jugador $B$ se enfrenta. Un jugador A con confianza social asignará una probabilidad $\theta>c / b+c$ de que $B$ sea digno de confianza, y por tanto optará por cooperar con B.

Una vez determinada la estructura de la confianza social, sigue pendiente la cuestión del origen de estas expectativas. ¿Cómo se crea la confianza social? Para el jugador $B$ puede resultar tentador actuar como un oportunista y engañar al jugador $\mathrm{A}, \mathrm{y}$, a su vez, $\mathrm{y}$ dada la estructura del juego de la confianza, es racional que $A$ piense que lo más probable es que $B$ sea un oportunista en el que no vale la pena confiar, o que, en cualquier caso, decida no arriesgarse.

La primera respuesta que encontramos en la literatura de capital social "es la creación de confianza social como subproducto de la realización de otras actividades, particularmente participación en asociaciones. Desgraciadamente, esta respuesta carece de microfundamentos. Los mecanismos que vinculan confianza con participación en asociaciones generalmente no están identificados, o, si lo están, tienden a ser débiles o triviales. Sugeriré a continuación varios mecanismos para esta relación. Después me ocuparé de la creación de confianza social empleando señales.

\section{CREACIÓN DE CONFIANZA SOCIAL COMO SUBPRODUCTO DE LA REALIZACIÓN DE OTRA ACTIVIDAD}

La creación de formas de capital social como subproducto de la realización de otras actividades es una idea presentada por James Coleman (1990). En el caso de la confianza social, la actividad principal a partir de la cual suele considerarse que se crea confianza social como subproducto es la participación en asociaciones. En Making Democracy Work, Robert Putnam (1993) consideraba que una de las vías a través de las cuales la confianza particularizada podía transformarse en confianza social era mediante la participación en redes de compromiso cívico. Estas redes de compromiso cívico se refieren a la participación en todo tipo de asociaciones voluntarias, desde sociedades ornitológicas a clubes de fútbol, y no meramente a aquellas dirigidas al ámbito político. Recoge aquí Putnam, conscientemente, la idea de Tocqueville del papel desempeñado por las asociaciones civiles a la hora de "engrandecer el corazón y desarrollar el espíritu humano" (Tocqueville, 1996: 98). El problema con esta fuente de creación de confianza social es que el mecanismo entre participación en asociaciones como ésas y la confianza en personas ajenas a las asociaciones no está nada claro (Levi, 1996a: 47-48). Es decir, no se sabe por qué el miembro de una peña de fútbol, por el hecho de pertenecer 
RIS

REVISTA INTERNACIONAL DE SOCIOLOGIA

№ 35, Mayo-Agosto, 2003

FRANCISCO HERREROS VÁZQUEZ

a esa asociación, va a tener una predisposición superior a la media a confiar en desconocidos. Presentaré cinco argumentos al respecto:

1. El primero se refiere a la percepción individual del "tipo" del resto de la población. Una persona confía en desconocidos porque ha tenido buenas experiencias con sus compañeros de asociación y considera que esos compañeros son una muestra representativa de la sociedad.

2. El segundo argumento considera que la participación en asociaciones es una forma de identificar propiedades inherentes a personas dignas de confianza.

3. El tercero considera los efectos de la deliberación en el seno de las asociaciones sobre las creencias de los participantes acerca de personas desconocidas.

4. El cuarto argumento es una derivación del tercero. Afirma que los efectos de la deliberación tienden a variar de acuerdo con el objetivo de la asociación.

5. El quinto considera que la forma y el número de las redes sociales a las que pertenece el individuo afectan al desarrollo de la confianza en personas desconocidas.

El primer argumento afirma que los miembros de la asociación son considerados por el individuo como una muestra representativa de la sociedad. Es decir, el miembro de una asociación forma expectativas acerca del comportamiento futuro de otros miembros utilizando la información que ha adquirido de experiencias pasadas con ellos. Entonces, estas expectativas son extrapoladas a la gente en general. Por ejemplo, si alguien ha tenido buenas experiencias con otros participantes en una asociación, es más probable que asigne una mayor expectativa de no ser dignos de confianza a los no miembros. La idea de que los miembros de una asociación son una muestra representativa de la sociedad se ve acrecentada, igualmente, si el grado de heterogeneidad en la asociación es alto, digamos en términos de ideologías, de gustos, o de origen étnico (Stolle y Rochon, 1998: 49). En asociaciones "inclusivas", como las denomina Putnam, por ejemplo las pertenecientes al movimiento de los derechos civiles, que incorporan personas separadas por diferentes fracturas sociales, es más sencillo el desarrollo de la confianza social (Putnam, 2000: 22) En todo caso, estas creencias acerca del comportamiento de otros individuos son difícilmente racionales ${ }^{1}$. Los miembros de una asociación dada claramente no son una muestra representativa de los seres humanos, y en ese sentido la extrapolación a extraños de su comportamiento no parece bien fundamentada. Si una muestra es muy grande, sin duda será altamente representativa de la población de la que ha sido extraída. Pero no ocurre lo mismo con las muestras pequeñas, como las que pueden representar la casi totalidad de

\footnotetext{
${ }^{1}$ En realidad, es dificil que unas creencias de este tipo superen siquiera el requisito de consistencia de la teoría estricta de la racionalidad. A decir de Elster (1983), la consistencia de las creencias requeriría, entre otras cosas, que sean conformes con las leyes de la probabilidad.
} 
las asociaciones, que, además, no son en ningún caso muestras aleatorias. No obstante, puede ser que detrás de este argumento haya algo de verdad. Si bien individuos perfectamente racionales no pueden creer que su asociación de amigos de las iguanas sea una muestra representativa de la sociedad, quizá la cosa cambie si relajamos el supuesto y asumimos que los individuos son sólo limitadamente racionales. Esta racionalidad limitada puede referirse a la capacidad computacional de los humanos, o a la cantidad de información que pueden plausiblemente adquirir, por ejemplo, sobre las estrategias de otros agentes. Esto se combina con la complejidad del entorno para explicar las percepciones subjetivas de la realidad realizadas por los humanos (Williamson, 1985: 45-46; North, 1990: 25). Por lo tanto, agentes limitadamente racionales se enfrentan a la incertidumbre acerca de las preferencias de personas desconocidas, tienen que asumir costes relativamente altos de reunir y procesar información sobre esas preferencias, y es bastante razonable pensar que el problema de representatividad de las muestras es suficientemente complejo para que se alcancen normalmente conclusiones erróneas al respecto. En esas circunstancias, ¿cómo puede el individuo formar sus expectativas? Una solución es emplear algún tipo de regla, como "si mis compañeros de asociación son dignos de confianza, consideraré que los desconocidos también lo son". La participación en asociaciones, por lo tanto, puede proporcionar a la gente una herramienta para juzgar si la gente es digna de confianza o no, $y$, en este sentido, puede promover la confianza social. La psicología cognitiva proporciona evidencia para apoyar este argumento. Numerosos experimentos realizados acerca de esta misma cuestión desde esta disciplina permiten llegar a la conclusión de que la gente tiene fuertes intuiciones, equivocadas en aspectos fundamentales, acerca de las muestras aleatorias. En general, parece que se cree que las muestras, cualquiera que sea su tamaño, son muy similares unas a otras y a la población, y que el muestreo es un proceso que se "auto corrige", de manera que toda desviación en un sentido con respecto a la media de la población se ve compensada con una desviación similar en sentido contrario. Es lo que Tversky y Kahneman (1986) denominan la "ley de los números pequeños": la ley de los grandes números se aplica también a números pequeños, lo que confirmaría la validez de conclusiones basadas en muestras pequeñas.

El segundo argumento considera que la participación en asociaciones es una forma de aprender a cómo leer señales asociadas con ser digno de confianza. Si hemos desarrollado relaciones de confianza con miembros de un grupo de individuos, o si conocemos algunas características de la cultura, las tradiciones o los valores de ese grupo, entonces podemos generalizar esa confianza a otra gente que tenga algún signo externo de pertenencia a ese grupo de individuos. Este grupo puede ser una comunidad étnica, por ejemplo (Bacharach y Gambetta, 1997; Offe, 1999: 63; Hardin, 1995; Blackburn, 1998), o una "comunidad imaginaria" como la nación (Whiteley, 1999: 31). En este caso la forma de reducción de costes de transacción es asociar a determinados rasgos visibles (por ejemplo, 
RIS

REVISTA INTERNACIONAL DE SOCIOLOGIA

№ 35, May0-Agosto, 2003

FRANCISCO HERREROS VÁZQUEZ

el color de piel o la forma de vestir) determinadas características (por ejemplo, la honradez) que son consideradas relevantes a la hora de formar expectativas acerca de si alguien es o no digno de confianza. Es lo que Bacharach y Gambetta (2001) denominan "propiedades que garantizan la confianza". En este sentido, la participación en redes constituiría una forma de aprender rasgos asociados con ser digno de confianza que también sirve para formar expectativas acerca de lo dignos de confianza que son desconocidos. Por tanto, esto sería la antesala de la "confianza en señales" de Bacharach y Gambetta (2001), el aprendizaje de qué señales están vinculadas a "propiedades que garantizan la confianza", como por ejemplo la honestidad. Esta forma de aprendizaje acerca del "tipo" de las personas dignas de confianza es distinta a la definición de Yamagishi (2001) de la confianza social como una forma de "inteligencia social". De acuerdo con Yamagishi, los que confian en los demás son personas que, enfrentados a altos costes de oportunidad y altos niveles de incertidumbre social, realizan fuertes inversiones en recursos cognitivos, como por ejemplo atención y memoria. Los que confian en los demás, de acuerdo con esto, asumen riesgos para aprender más acerca de lo dignos de confianza que son los demás. De acuerdo con mi argumento, los que confían en los demás lo hacen porque han aprendido en el seno de su asociación qué signos están ligados a ser digno de confianza. También se enfrentan a incertidumbre, pero las probabilidades subjetivas que se forman acerca de lo dignos de confianza que son los demás están mejor fundamentadas gracias a su proceso de aprendizaje dentro de las asociaciones.

El tercer argumento sobre la relación entre participación en asociaciones y confianza social considera que la participación en asociaciones puede transformar las creencias de sus integrantes a través de la deliberación. En primer lugar, ciertas características del proceso de deliberación podrían conducir a un cambio de preferencias. Una de estas características puede ser las normas del proceso. A menudo se argumenta que si la discusión es pública, hay una presión para abstenerse de argumentos egoístas (Elster, 1995: 390). Se afirma que hay un deseo por parte de los participantes de no parecer egoístas, porque esto sería embarazoso o vergonzoso (Fearon, 1998: 54). Otro posible mecanismo para explicar por qué los participantes en un proceso deliberativo generalmente justifican sus puntos de vista en términos del bien común es el mecanismo psicológico de la reducción de la disonancia cognitiva: los individuos tienden a hacer coincidir lo que hacen con lo que piensan, para reducir disonancia (Elster, 1987: 113). Otra característica de la deliberación que puede llevar a un cambio de preferencias es que la deliberación puede revelar información privada (Fearon, 1998: 46; Gambetta, 1998: 22). Algunos participantes pueden reconsiderar sus preferencias dada la nueva información ahora disponible. Estos tres efectos de la deliberación pueden relacionarse con la creación de confianza social. En primer lugar, la discusión pública lleva al individuo a presentar sus argumentos en términos del bien común. Entonces actúa el segundo efecto: reduciendo disonancia haciendo concordar lo que la gente 
piensa con lo que hacen. El tercer efecto, la reunión de información acerca de otros participantes, es independiente de los otros dos. Un ejemplo podría ilustrar cómo estos efectos de la deliberación se relacionan con la confianza social. Este ejemplo esta tomado de las "encuestas deliberativas" de James Fishkin y Robert Luskin (2000: 25). Al comienzo de una de estas encuestas, un conservador blanco de 84 años de Arizona cuestionó si uno de los miembros del grupo, una madre soltera afroamericana de Nueva York que recibía ayuda pública, tenía realmente una familia, dado que, en su opinión, una familia requería una madre, un padre e hijos. Al final de la reunión de dos días, admitió que se había equivocado. En este ejemplo pueden operar dos mecanismos. El conservador blanco pudo cambiar sus creencias acerca de las madres solteras afroamericanas que viven gracias a la ayuda pública ( $\mathrm{y}$, quizás, acerca de los neoyorquinos) porque tenía que evitar comentarios demasiado hostiles sobre los aforamericanos, para evitar sufrir la sanción del grupo. $O$ simplemente puede que aprendiese más acerca de las madres solteras afroamericanas que le llevase a pensar que, después de todo, se podía confiar en ellas.

¿Se dan estos efectos de la deliberación sobre las creencias de los participantes con el mismo grado en todos los tipos de asociaciones? De acuerdo con Putnam, sí. $^{2}$ Otros autores, como Stolle y Rochon (1998) han mostrado que diferentes tipos de asociaciones tienen distintos efectos sobre la generación de confianza social. Consideran que las asociaciones culturales y comunitarias están fuertemente ligadas al desarrollo de confianza generalizada.

El cuarto argumento de los presentados más arriba afirma que los objetivos de las asociaciones tienen efectos sobre la deliberación. Distinguiré entre asociaciones civiles y políticas. Esta distinción está tomada de Tocqueville. De acuerdo con Tocqueville (aunque en esta cuestión, como en otras de La democracia en América no es del todo consistente), el desarrollo de asociaciones políticas es más sencillo que el desarrollo de asociaciones civiles. Por un lado, la participación en asociaciones civiles es más costoso: en la mayor parte de ellas, los miembros tienen que arriesgar parte de su dinero (parece que Tocqueville estaba pensando en asociaciones comerciales e industriales). Por otro lado, los beneficios de las asociaciones parecen mayores en la vida política que en la civil. En la vida civil las personas son consideradas más autónomas, mientras que en la vida política la necesidad de cooperar para alcanzar objetivos comunes es más obvia (Tocqueville, 1995: 102-107). Para Tocqueville las asociaciones civiles parecen aquéllas constituidas para obtener un bien privado, mientras que las asociaciones públicas se constituyen para obtener un bien colectivo, que en ciertos casos puede además

\footnotetext{
${ }^{2}$ Aunque Putnam considera que cierto tipo de asociaciones, las verticales, no son propicias para el desarrollo de virtud cívica, también considera que el desarrollo de preferencias cívicas es independiente del objetivo de la asociación: no importan si la asociación es política o no.
} 
RIS

ser un bien público. Aunque la deliberación como discusión puede tener lugar en ambos tipos de asociaciones, desde el punto de vista de la deliberación como una forma de desarrollar confianza social, las asociaciones políticas parecen más interesantes. Éste, al menos, parece ser el caso si consideramos el contenido de la discusión en ambos tipos de asociaciones. Algunos de los efectos de la deliberación, como la revelación de información privada o la superación de la racionalidad limitada (Fearon, 1998), pueden tener lugar tanto en asociaciones civiles como en políticas. No obstante, la transformación de creencias en confianza social parece más probable si el contenido de la discusión es política. Esto se debe a mecanismos tales como la formulación de preferencias en términos del bien común debido a la presión de grupo, y los cambios de preferencias y creencias para reducir disonancia cognitiva. Mi idea es que en las asociaciones políticas, las presiones para formular preferencias y creencias en términos del bien común incluye no sólo el bien de los miembros de la asociación, sino, debido a la naturaleza misma de la asociación, el bien común de la comunidad en sentido amplio.

El quinto argumento acerca de la relación entre participación en asociaciones y generación de confianza social afirma que la participación en asociaciones también puede promover la confianza en no miembros porque las pérdidas potenciales de aquel que confía en el caso de que el depositario de la confianza no sea digno de ella disminuyen si aquel que confía puede basarse en sus redes sociales. Si soy miembro de varias asociaciones, y he desarrollado relaciones de confianza particularizada, puedo confiar en desconocidos incluso si desconozco cuál es su tipo, porque puedo compensar las pérdidas potenciales de ser engañado basándome en mis redes sociales. La decisión de confiar es una decisión racional basada en tres consideraciones: la probabilidad subjetiva que se le asigna al otro individuo de ser digno de confianza, las pérdidas potenciales en el caso de que no sea digno de confianza, y las ganancias potenciales en el caso de que haga honor a la confianza que en él se ha depositado. Teniendo en cuenta estos tres elementos, un individuo decidirá confiar en otro siempre que $p G>(1-p) L, 0$, lo que es lo mismo, cuando $p / 1-p>L / G$, donde $p=$ probabilidad de que el otro jugador sea digno de confianza, $L=$ pérdida potencial y $G=$ ganancia potencial

Figura 4.

Red social y confianza social

(a)


(Coleman, 1990: 99). La posesión de una determinada red social puede reducir el cociente $L / G$ de dos maneras. En la figura 4, por un lado, se puede argumentar que las pérdidas para $A$ de ser engañado por $E$ si deposita su confianza en él serán percibidas como potencialmente menores en este caso porque podrá basarse en B y D para restañar sus pérdidas. En sí este argumento no afirma nada más que aquel que tiene más recursos experimentará unas pérdidas potenciales comparativamente menores que aquel que tiene menos, y por tanto, tenderá a confiar más, manteniendo todo lo demás constante (una persona con muchos recursos puede, a pesar de todo, ser sumamente desconfiada debido a que otras variables de la ecuación, como $p$, tengan un valor muy bajo) Dado que el capital social es al fin y al cabo un recurso, ese argumento también se aplica a este caso. Un argumento algo distinto relaciona la posesión de redes sociales con otra de las variables de la ecuación, en esta ocasión $\mathrm{G}$, las ganancias potenciales. Volviendo a la figura 4, en el caso de que $\mathrm{E}$ responda favorablemente a la confianza que $\mathrm{A}$ ha depositado en él, esto se traduce en una ganancia para $\mathrm{A}$. Añade a $\mathrm{E}$ a su relación de amigos o conocidos, y, como consecuencia, añade recursos potenciales de capital social que quizá pueda movilizar en un futuro. Ahora supongamos que a estas relaciones A añade sucesivamente varias docenas más, por su pertenencia a muchas más redes sociales, o bien porque su red social original se amplía extraordinariamente. $\mathrm{Al}$ aumentar su número de amigos, podría considerarse que la utilidad marginal de cada nueva amistad es cada vez menor, es decir, que las ventajas que supone para A aumentar su número de amigos son cada vez menores. Comparemos esta situación con la opuesta, la de un individuo solitario. Un habitante del estado de naturaleza de Hobbes, donde la vida es "solitaria, pobre, desagradable, brutal y corta" (Hobbes, 1992: 108) valoraría sin duda mucho más contar con un amigo. Es decir, que si bien para $A$ la variable $G$ tiene un valor muy pequeño, para un individuo solitario $\mathrm{G}$ es muy grande. No obstante, mi idea no es que la participación en redes sociales favorezca la cooperación debido a que incremente la ratio $p /(1-p)$, es decir, porque aumente la confianza social. Las expectativas acerca de lo dignas de confianza que son las personas pueden cambiar a través de un proceso de "racionalización de la esperanza". Como he dicho, en este caso los beneficios potenciales $(\mathrm{G})$ de entrar en una nueva relación son muy altos, y esto hace que la decisión de cooperar con personas desconocidas sea más probable. No obstante, los costes de confiar en individuos que no son dignos de confianza pueden ser especialmente altos en este caso. En la novela clásica de Thomas Hardy Tess of the D'Urvervilles la protagonista decide confiar en su supuesto primo Alec, con la consecuencia previsible en toda buena novela naturalista de acabar siendo violada. El resultado es más terrible aún debido a que la pobre Tess carece prácticamente de amigos en quienes buscar consuelo y su familia sería clasificada en un análisis actual de capital social como francamente desestructurada. Dado que las pérdidas que se experimentan en estos casos de carencia de redes sociales en las que basarse pueden ser especialmente crudas, puede ocurrir que las expectativas sean conformadas 
RIS

REVISTA INTERNACIONAL DE SOCIOLOCIÁ

№ 35, May0-Agosto, 2003

FRANCISCO HERREROS VÁZOUEZ

en un sentido favorable a ciertos estados del mundo que prefiero que ocurran por encima de estados del mundo que falsearían esas expectativas. La consecuencia, por tanto, sería una expectativa $p$ mayor, y, por lo tanto, una probabilidad mayor de confiar en desconocidos, manteniendo constantes las demás variables.

El resultado es un tanto paradójico para el argumento de que la participación en asociaciones genera más confianza social. Este argumento, por el contrario, afirma que cuanto más grandes son tus recursos de capital social, por ejemplo porque participas en muchas asociaciones, menor es el beneficio que experimentas confiando en desconocidos. En realidad, este resultado no es tan extraño. Detrás de la idea, planteada también por Putnam, de que determinadas asociaciones son autosuficientes y excluyentes (Putnam, 2000: 22), puede estar en realidad este argumento, aunque en esta ocasión aplicado a cualquier tipo de asociaciones. El argumento que acabo de desarrollar no implica, en todo caso, que cuanto mayor sea el número de asociaciones a las que se pertenezca, menor vaya a ser el capital social. Lo que implica es que los beneficios marginales de obtener nuevas relaciones personales son decrecientes, y esto debería tener impacto sobre la decisión de confiar en desconocidos. Dado que, se supone, la participación en asociaciones favorece la confianza en desconocidos en la medida en que los miembros de tu asociación sean considerados una muestra representativa de la sociedad, es de esperar que a cuantas más asociaciones se pertenezca, mayor será tu muestra, y mayor la probabilidad de confiar en desconocidos. No obstante, ya que los beneficios marginales de confiar en desconocidos son decrecientes, a partir de un punto puede ser que, perteneciendo a muchas asociaciones, tu probabilidad de confiar en desconocidos comience a disminuir. La relación entre número de asociaciones a las que se pertenece y probabilidad de confiar en desconocidos tendría la forma de la figura 5.

Figura 5.

Número de asociaciones y confianza social

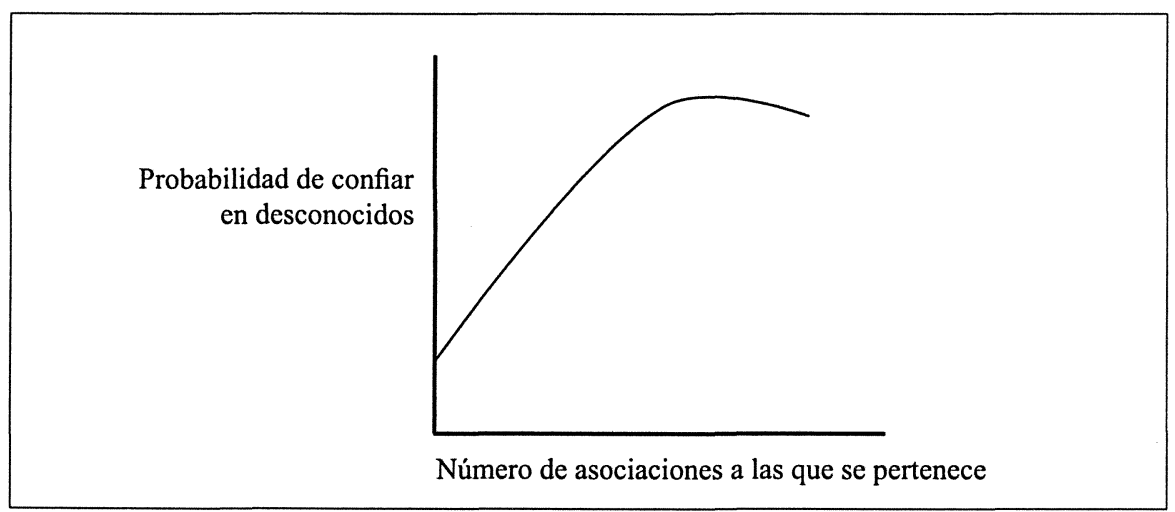


De manera que la red social a la que se pertenezca puede tener dos efectos sobre la generación de confianza social. Por un lado, formar parte de una red social puede conferir recursos que hagan más probable que se corran riesgos. Pero, por otro, si la red social es muy amplia, puede que el beneficio marginal de hacer nuevas relaciones disminuya, por lo que individuos que, por ejemplo, participen en muchas asociaciones, puede que desplieguen menos confianza social.

He propuesto, por lo tanto, cinco mecanismos para la relación entre participación en asociaciones y confianza social. A continuación me referiré a otra fuente de creación de confianza social: su creación mediante señales.

\section{LAS SEÑALES COMO FUENTE DE CONFIANZA SOCIAL}

En la anterior discusión a propósito de los distintos mecanismos implicadós en la creación de confianza social como subproducto de la participación en asociaciones mencioné que la pertenencia a asociaciones, podría ser considerada una forma de aprender ciertas señales asociadas con ser digno de confianza. En esta sección, consideraré este tema desde un ángulo opuesto. Discutiré cómo alguien puede inducir a otras personas a confiar en él lanzando ciertas señales de que es digno de confianza.

La estructura de esta forma de creación de confianza social se recoge en la figura 6. El juego de la figura 6 es el juego de la confianza como un juego de señales. Este juego de señales, al igual que el juego de la confianza social de la figura 3, es un juego de información imperfecta y asimétrica. En este tipo de juegos, todos o algunos de los jugadores tienen información privada que sólo ellos conocen. La información privada de cada jugador determina su tipo. Al igual que en el juego de la confianza social, uno de los jugadores, en este caso el jugador A, puede ser de dos tipos: digno de confianza u oportunista. No obstante, a diferencia del juego de la confianza social, en este caso el jugador A puede comunicar su información privada mediante una señal al jugador $B$ antes de que $B$ decida qué hacer. En este caso, la señal enviada por el jugador A sirve para que el jugador $\mathrm{B}$ revise sus expectativas acerca de si A es o no digno de confianza. Es decir, un individuo puede emplear conscientemente determinados signos externos que sepa que son asociados a características, como por ejemplo la honestidad, que se consideran propias de una persona digna de confianza, lo que Bacharach y Gambetta (1997) denominan "propiedades que garantizan la confianza". En la novela de Truman Capote $A$ sangre fría, los dos protagonistas deciden cambiar de aspecto, afeitarse y ponerse trajes nuevos antes de pasar cheques sin fondo en varias tiendas de Kansas City. Los dueños estafados declararon después que ambos inspiraban confianza porque iban bien vestidos y hablaban bien. En El Príncipe, Maquiavelo sostiene que los ciudadanos, al no poder conocer personalmente a su gobernante, deben confiar en él basándose sólo en signos externos y apariencias, 
RIS

REVISTA INTERNACIONAL DE SOCIOLOCIA

Figura 6.

El juego de la confianza como un juego de señales

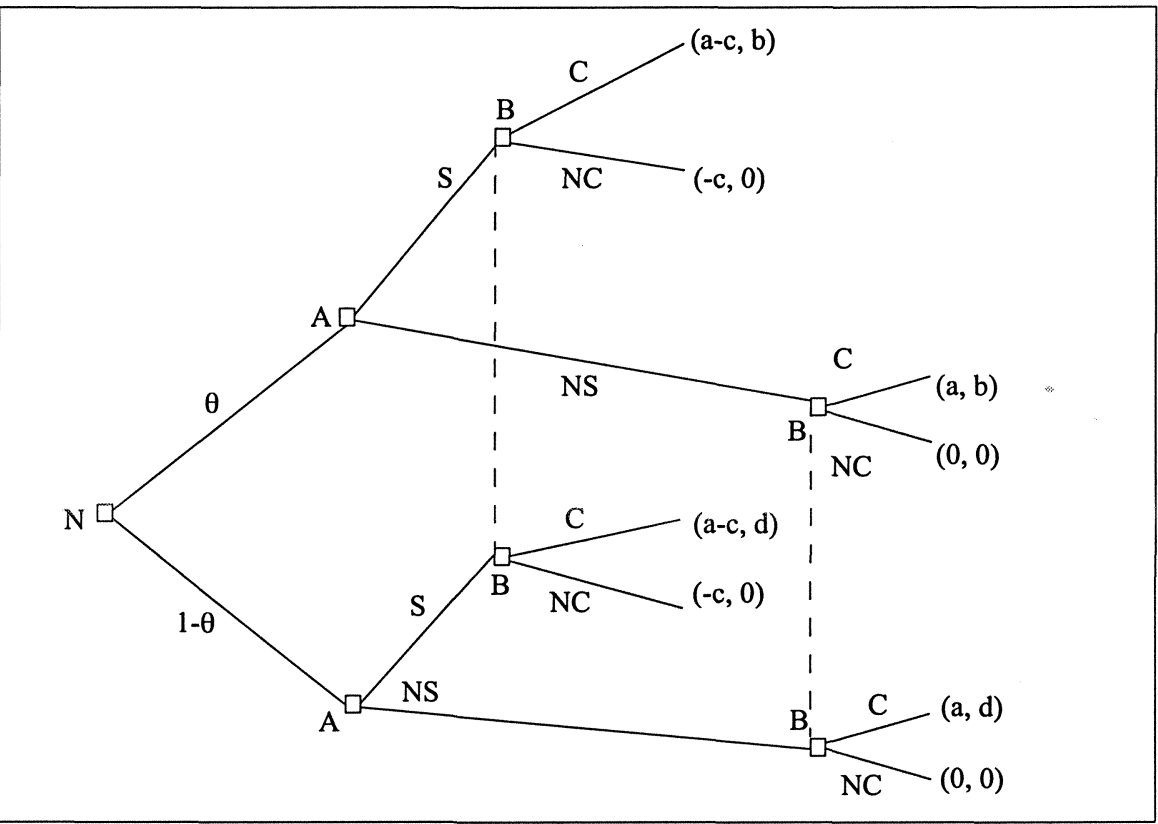

Movimientos del jugador A:

$S=$ enviar una señal

$\mathrm{NS}=$ no enviar una señal

Movimientos del jugador B:

$\mathrm{C}=$ Cooperar

$\mathrm{NC}=$ no cooperar

$\theta=$ probabilidad de ser digno de confianza

1- $\theta=$ probabilidad de no ser digno de confianza

algo que, de hecho, favorece que el príncipe se comporte de forma oportunista (Maquiavelo, 1992: 92). No es necesario, claro está, que aquel que envía la señal sea un oportunista. Puede que sus intenciones sean buenas, señalar al otro que es digno de confianza cuando en realidad lo es.

En el juego de la figura 6, el jugador A tiene dos opciones: lanzar una señal al jugador B (S) o no lanzarla (NS). Lanzar una señal supone un coste, $c$, que se resta a los pagos del jugador A, siempre que este lance una señal. En el caso de que $\mathrm{B}$ decida cooperar con él, recibirá como pago $a$. Si B decide no cooperar con él, recibe un pago de 0 . El orden de sus pagos será: $a>a-c>0>-c$, asumiendo 
que los beneficios derivados de obtener la confianza de B superen los costes de enviar la señal. El jugador B, por su parte, tiene también dos opciones, cooperar con $\mathrm{A}(\mathrm{C})$ o no cooperar (NC). Recibe un pago $b$ en el caso de que coopere con A y este resulte ser digno de confianza, un pago de $d$ si deposita su confianza en un jugador A oportunista, y 0 si decide no cooperar. El orden de sus pagos es, en consecuencia: $b>0>d$. En este juego se pueden alcanzar varios equilibrios. En uno de ellos, el jugador $B$ acaba confiando porque la señal que recibe hace que revise sus creencias iniciales acerca del tipo del jugador A. Se trata del siguiente equilibrio bayesiano perfecto: (S/Digno de confianza, NS/Oportunista; C, NC: 1, 0). Es decir, si el jugador A es digno de confianza, lanza una señal de que lo es. Si es un oportunista, no lanza esa señal. El jugador B sólo confía en este caso si el jugador A lanza su señal. En el caso de que ambos tipos de jugadores, el digno de confianza y el oportunista, decidan lanzar señales, las señales no añaden ninguna información para el jugador B. En este caso, un equilibrio posible es: (S/Digno de confianza, S/Oportunista; $C, C: \theta, 0) \operatorname{con} \theta>\mathrm{d} / \mathrm{d}-\mathrm{b}$. De manera que, dados los pagos del juego de la figura 5 , sólo si el jugador $B$ considera inicialmente que la probabilidad de que A sea digno de confianza es mayor que $\mathrm{d} / \mathrm{d}-\mathrm{b}$, decidirá cooperar.

Por lo tanto, en el juego de la figura 6 , el jugador A puede realizar acciones que lleven al jugador B a confiar en él. El jugador B tiene inicialmente una creencia sobre lo digno de confianza que es A. Esta creencia puede ser superior o inferior a d/d-b (dados los pagos de este juego), y llevarle o no, en consecuencia, a confiar. En puridad, el jugador A crea confianza social, comunicando al jugador B una información clave para que este revise sus creencias iniciales y pase, así, a confiar en un desconocido del que carece, por lo demás, de información acerca de cómo se ha comportado en el pasado. Fundamentar las propias expectativas en señales externas puede no ser la mejor forma de configurar creencias. No obstante, es algo que puede ser empleado por el que lanza las señales para generar confianza en sí mismo (quizá, manipulando al otro).

A continuación presentaré un ejemplo que ilustra esta idea de que la confianza en señales puede ser analizada de manera fructífera desde el lado de la oferta, es decir, como una fuente de confianza social ${ }^{3}$.

Las comunidades campesinas en Vietnam no se caracterizaban precisamente por la confianza generalizada entre sus miembros. Esto puede resultar curioso, ya que normalmente se asume que las pequeñas comunidades rurales son especialmente proclives al desarrollo de relaciones de confianza. Especialmente significativo en ese sentido es el trabajo de Michael Taylor acerca de la superación de dilemas de acción colectiva en las pequeñas comunidades campesinas (Taylor, 1988). Este

\footnotetext{
${ }^{3} \mathrm{La}$ mayoría de las referencias a las comunidades campesinas vietnamitas y a las actividades de los empresarios políticos están tomadas del libro de Samuel Popkin (1979).
} 
R I S

REVISTA INTERNACIONAL DE SOCIOLOCIA

No 35, Mayo-Agosto, 2003

trabajo de Taylor estaba deliberadamente dirigido a suministrar microfundamentos desde la teoría de la elección racional a las afirmaciones de Theda Skocpol en Los Estados y las revoluciones sociales acerca de la actividad revolucionaria de las comunidades campesinas de Francia, Rusia, y China.

Las aldeas vietnamitas eran, sin duda, comunidades pequeñas, de entre $300 \mathrm{y}$ 1000 personas. Las familias se agrupaban por "barrios". Cada aldea contaba con un jefe, un consejo de notables, y un sistema de rangos para determinar la jerarquía social. La mayor parte de la tierra, las tres cuartas partes del total, era propiedad privada de las familias campesinas. El resto, la cuarta parte, era tierra comunal (al menos en las provincias de Tonkín y Annam), administrada y controlada por el consejo y el jefe de la aldea.

Las aldeas vietnamitas se caracterizaban por la contraposición de intereses entre sus miembros, y, como consecuencia, por una profunda desconfianza que dificultaba la solución de muchos de los dilemas sociales a los que se enfrentaban los campesinos. Los recursos de la aldea eran escasos, y esto originaba competencia y tensiones entre los campesinos.

La principal desconfianza iba dirigida hacia los notables que gobernaban la aldea. Esta situación de desconfianza es un ejemplo de las dificultades para el desarrollo de relaciones de confianza en organizaciones verticales. Como ya vimos, para Putnam (1993a), las relaciones verticales son un obstáculo para el desarrollo de relaciones de confianza debido a que existen incentivos, tanto desde el punto de vista de los superiores jerárquicos como de sus subordinados, para comportarse de forma oportunista. Este oportunismo se sustancia en la explotación de los subordinados por sus superiores, y en la desviación de responsabilidades por parte de los primeros. Una forma de evitar estos obstáculos al desarrollo de relaciones de confianza es proporcionar reglas institucionales fijas que establezcan con claridad las acciones de cada una de las partes, y sancionen su incumplimiento. Se trata de una solución habitual a los problemas de asimetrías informativas en organizaciones jerárquicas (Miller, 1992: 225) En todo caso, estas reglas institucionales fijas no solucionaban los problemas de desconfianza en las aldeas vietnamitas porque, de hecho, sancionaban y reforzaban las desigualdades en riqueza existentes. Las normas fijas establecían que los recursos fueran asignados entre los campesinos por su rango en la aldea, y el rango era determinado en gran medida por la edad, la riqueza y la educación. Como consecuencia, los notables veían reforzada su posición, e igualmente sus incentivos para defraudar a los campesinos más pobres. Y, de hecho, aprovechaban su posición de privilegio para obtener ventajas frente a los campesinos. Al servir en el consejo de notables, los más ricos de la aldea tenían acceso a los listados de la aldea y los registros de los mandarines. El número de aldeanos inscritos en el registro de los mandarines determinaba la cuantía de los impuestos que tenía que pagar la aldea. De manera que el interés colectivo de la aldea demandaba que el número de inscritos en el registro de los mandarines fuese el menor posible. No obstante, el interés individual de los notables les llevaba a 
querer estar inscritos en los registros. Estar inscritos en esos registros suponía, de hecho, poder acceder al consejo de notables, poder acudir a los tribunales, pedir la ayuda de los mandarines, o viajar con seguridad fuera de la aldea. Estos beneficios eran utilizados por los notables para reforzar su situación. Y, aunque en teoría sólo aquellos que estuviesen inscritos en el registro de los mandarines tenían la obligación de pagar impuestos, en la práctica el impuesto a pagar por la aldea era compartido por todos los miembros de la misma a partes iguales.

La desconfianza y la envidia también eran actitudes generalizadas entre los campesinos pobres de las aldeas vietnamitas. Dada la escasez de recursos y la competencia por los mismos, los campesinos percibían la vida como un juego de suma cero, por lo que consideraban que era mejor la no cooperación, ya que cooperar implicaba el riesgo de que tu vecino adquiriese una ventaja a tu costa. En todo caso, la confianza sólo surgía en relaciones horizontales, entre miembros de la misma familia, conocidos y amigos. Esas limitadas relaciones de confianza no se extendían más allá de la aldea: hacia los extraños existía, por lo general, una profunda desconfianza. Es decir, los aldeanos vietnamitas no se distinguían por sus fuertes expectativas de confianza generalizada. Además, era esperable que esta desconfianza fuera mayor entre los pobres que entre los ricos, dado que estos últimos tenían más recursos y experimentarían unas pérdidas potenciales menores en el caso de ser engañados. Efectivamente, parece que las relaciones sociales en Cochinchina, la más rica de las tres provincias de Vietnam, se caracterizaban por niveles mayores de confianza.

Entre los mismos notables estaba presente una actitud normal de desconfianza. Esto se reflejaba en la norma de decisión en el consejo: en las aldeas vietnamitas las decisiones se tomaban por unanimidad en el consejo de notables. Esto, más que reflejar un consenso subyacente entre los más ricos de la aldea, reflejaba más bien las relaciones básicas de desconfianza entre ellos. La unanimidad suponía que todos ellos tenían poder de veto sobre cualquier decisión que lesionase sus intereses. De hecho, cuando un notable podía aprovechar cualquier influencia externa para mejorar su posición frente a los demás, lo hacía. Durante la dominación francesa, el jefe de la aldea empleaba sus relaciones con las autoridades coloniales para socavar el poder del resto de notables.

Dada esta situación de desconfianza generalizada entre campesinos y notables, por un lado, y dentro de cada categoría, por otro, era difícil la realización de algunos trabajos que requiriesen un cierto grado de cooperación. No obstante, esto no quiere decir que no existiese cooperación. Existían, de hecho, asociaciones formadas por pequeños grupos de campesinos. Por ejemplo, asociaciones de vecinos para compartir los gastos de las fiestas comunitarias, asociaciones para compartir los costes de comprar cerdos, asociaciones para compartir los costes de las ceremonias religiosas. En todos esos casos, la cooperación se producía, pero no gracias a la presencia de relaciones de confianza. La participación en algunas de esas asociaciones no requería la existencia de relaciones de confianza, ya que 
RIS

REVISTA INTERNACIONAL DE SOCIOLOGIA

№ 35, Mayo-Agosto, 2003

FRANCISCO HERREROS VÁZQUEZ

su objetivo no eran generalmente bienes públicos, sino privados. Las asociaciones de reparto de costes para comprar cerdos, por ejemplo, sólo beneficiaban a aquel que pertenecía a la asociación. Simplemente, quien no perteneciese debía asumir él solo los costes de su compra. El objetivo de otras asociaciones quizá se asemejase más a un bien público. Por ejemplo, las asociaciones para asumir los costes de las celebraciones religiosas. Se puede pensar que las celebraciones religiosas reunían características de bien público, dado que beneficiaban tanto a quienes hubiesen colaborado a su organización como a quienes no. En este caso, las preferencias de los campesinos eran las propias de un juego de coordinación, no de un dilema del prisionero. Es decir, la primera preferencia de cada uno de ellos es que todos colaborasen en los preparativos de las fiestas, pero si los demás no están dispuestos a colaborar, la mejor respuesta es, a su vez, no colaborar. En una situación como ésta las expectativas acerca del comportamiento de los demás pueden resultar cruciales. Pero, como hemos visto, las aldeas vietnamitas no se caracterizaban por la omnipresencia de relaciones de confianza. En su ausencia, la solución a los dilemas sociales a los que se enfrentaban los campesinos suponía incurrir en costes de supervisión. Dado que las asociaciones incluían pocos miembros por lo general, estos costes de supervisión no eran demasiado altos, por lo que la cooperación podía mantenerse. No obstante, estos costes eran, en todo caso, superiores a aquellos en los que se hubiese incurrido si existiesen relaciones de confianza entre los campesinos.

En este entorno hostil al desarrollo de relaciones de confianza, inician sus actividades en las aldeas durante la época de la dominación colonial francesa empresarios políticos pertenecientes a distintas organizaciones: la iglesia católica, las sectas Cao Dai y Hoa Hao, y el Partido Comunista. La labor de estos empresarios políticos fue crucial para solucionar buena parte de los dilemas de acción colectiva a los que se enfrentaban los campesinos en las aldeas vietnamitas. De acuerdo con Popkin, estos resultados se conseguían por métodos tales como manipulación de la información para convencer a los campesinos de que su contribución era crucial para lograr un determinado objetivo, o, por ejemplo, dividiendo un objetivo global en objetivos más pequeños con umbrales críticos. Pero, más allá de las formas a través de las cuales los empresarios políticos resolvieron los dilemas de acción colectiva de los campesinos de las aldeas vietnamitas, queda por resolver la cuestión de cómo consiguieron la colaboración de los campesinos. El problema es relevante, ya que a primera vista esto no era una tarea sencilla en unas comunidades rurales dominadas por la desconfianza hacia los extraños.

Para intentar resolver esta cuestión recurriré al juego de la confianza como un juego de señales. En ese juego, uno de los jugadores lanzaba una señal que proporcionaba al otro jugador información acerca de lo digno de confianza que es. De esta manera, el jugador que lanza la señal puede contribuir a crear capital social en forma de confianza, que luego puede emplear para otros fines. 
Existe evidencia de que los empresarios políticos lanzaron diversas señales para ganarse la confianza de unos campesinos por lo general desconfiados. Un ejemplo es el empleo de un lenguaje similar al utilizado por los propios campesinos. Muchos empresarios políticos fracasaron por no emplear este lenguaje. En las aldeas de Tonkín y Annam, los primeros empresarios políticos comunistas y trostskistas, todos ellos habitantes de las ciudades y muchos educados en Europa o en las escuelas francesas de Vietnam (y cuyo conocimiento de los campesinos se reducía en muchos casos a la lectura de traducciones vietnamitas de obras francesas), fracasaron en su intento de reclutar campesinos para su causa. Por el contrario, los sacerdotes católicos desde el principio se comunicaban con los campesinos empleando su propio vocabulario y expresiones. Por un lado, esto facilitaba considerablemente la comunicación, pero además el emplear el lenguaje propio de los campesinos actuaba como una señal. En ocasiones, los comunistas recurrían para ganarse a los campesinos a las "historias cantadas", una forma de comunicación de noticias muy familiar entre los campesinos. Un ejemplo algo extravagante de esto es la historia elaborada por los cuadros del soviet de NgheTinh en 1930-1, titulada "El consejo de una mujer a su marido para que haga la revolución", donde se narraba de forma macarrónica el paso de Marx por París durante la revolución de 1848 (Woodside, 1976: 181). Otra señal que podía ser interpretada favorablemente por los campesinos era el celibato de los sacerdotes católicos. La señal equivalente lanzada por los miembros de la secta Cao Dai y los militantes del partido comunista era su ascetismo. Ambos vestían ropas que no eran muy distintas de las que utilizaban los propios campesinos, y seguían un estilo de vida similar al de los campesinos pobres de la comunidad. El propio Ho Chi Minh, el hijo de una elitista familia de la intelligentsia confuciana vietnamita, se dirigió en el verano de 1941 a los habitantes de varias aldeas de la región de Nung vestido como un campesino (Woodside, 1976: 222).

En muchos casos, las señales mandadas por los empresarios políticos eran intencionales. No lo era, desde luego, el celibato de los sacerdotes católicos, pero sí su empleo de las expresiones y el vocabulario propio de los campesinos. Igualmente, vestirse igual que los campesinos y comer como los campesinos pobres de la aldea eran señales conscientes enviadas por los empresarios políticos comunistas de que eran dignos de confianza. A menudo, estas señales eran enseñadas a los empresarios políticos en ciernes. Un ejemplo de ello se encuentra en la "Escuela Libre de Tonkín", una institución creada en Hanoi en 1907 por elites locales de la provincia, y que buscaba extender a los campesinos su ideario nacionalista. Una de las recomendaciones de la escuela a sus jóvenes estudiantes, normalmente hijos de mandarines, era que para ganarse al campesinado había que "abandonar las mangas anchas y las ropas amplias y llevar las mismas ropas que los campesinos" (Woodside, 1976: 41). Estas señales proporcionaban información a los campesinos sobre algunos rasgos básicos de los empresarios políticos. En concreto, la austeridad de los empresarios políticos, tanto comunistas como cató- 
RIS

REVISTA INTERNACIONAL DE SOCIOLOCIA

№ 35, May0-Agosto, 2003

FRANCISCO HERREROS VÁZQUEZ

licos y miembros de la secta Cao Dai podía ser interpretada por los campesinos vietnamitas como reflejo de unas preferencias no egoístas. Las afirmaciones de los empresarios políticos en el sentido de que su labor iba dirigida a mejorar la situación material de los campesinos, y no a su propio beneficio, resultaban más dignas de confianza dado que la austeridad que mostraban comunistas y activistas Cao Dai era muestra de unas preferencias más altruistas.

En este caso, los empresarios políticos ayudaban conscientemente a construir expectativas de confianza generalizada entre los campesinos. Como ya he dicho, las señales (al menos muchas de ellas) eran lanzadas intencionadamente, y mediante esas señales los campesinos revisaban sus creencias acerca de lo digno de confianza que era el empresario político. Sus expectativas iniciales dependían de muchos factores, como por ejemplo la extrapolación a los desconocidos de las relaciones observadas en la aldea. Si es cierto que esas extrapolaciones de hecho se hacen, en el caso de las aldeas vietnamitas, dominadas por la desconfianza, la extrapolación a los extraños llevaría a niveles bajos de confianza generalizada, como de hecho así era. Las señales lanzadas por los empresarios políticos hacían que los campesinos revisasen sus expectativas en un sentido más proclive a la confianza en desconocidos. Porque, de hecho, los campesinos no tenían información acerca de cuáles eran las preferencias de los militantes comunistas o los sacerdotes católicos. Si finalmente decidían confiar en ellos, esto podía deberse en gran medida a que sus expectativas acerca de la confianza en personas básicamente desconocidas habían sido modificadas por las señales mandadas por los empresarios políticos. En este sentido, se puede decir que los empresarios políticos ayudaban conscientemente a crear capital social en forma de confianza generalizada. Posteriormente, podían emplear esta confianza así creada para conseguir superar algunos de los dilemas sociales a los que se enfrentaban los campesinos, desde la construcción de diques para mejorar sus cultivos, hasta la lucha contra las autoridades coloniales francesas.

\section{CONCLUSIONES}

En este trabajo he propuesto dos vías a través de las cuales puede crearse confianza social, ese peculiar tipo de confianza dirigida a individuos de los que se carece de información. En primer lugar, como consecuencia de la participación en asociaciones, a través de cinco mecanismos: la consideración de los miembros de la asociación como una muestra representativa de la sociedad, la identificación de propiedades de personas dignas de confianza, los efectos de la deliberación sobre creencias y preferencias, los efectos del tipo de asociación sobre la deliberación, y, finalmente, por los efectos de la forma de la red social a la que se pertenece sobre los costes, los beneficios y las creencias asociadas a la decisión de cooperar. En segundo lugar, de forma directa, enviando señales que hagan que los depositarios de la confianza revisen sus creencias iniciales acerca de lo dignos de confianza que son los desconocidos. 


\section{REFERENCIAS BIBLIOGRÁFICAS}

AXELROD, R. (1984), The Evolution of Cooperation, New York, Basic Books.

BACHARACH, M. y D. GAMBETTA (1997), "Trust in Signs", Russell Sage Foundation Working Paper from the Working Group on the Construction and Maintenance of Trust.

BLACKBURN, S. (1998), "Trust, Cooperation, and Human Psychology", en V. Braithwaite y M. Levi (eds.), Trust and Governance, New York, Russell Sage Foundation.

COLEMAN, J. S. (1990), Foundations of Social Theory, Cambridge, Harvard University Press.

DE TOCQUEVILLE, A. (1996), La democracia en América, Madrid, Alianza Editorial.

ELSTER, J. (1983), Uvas amargas, Barcelona, Península.

(1985), Making Sense of Marx, Cambridge, Cambridge University Press.

(1987), "The market and the forum: three varieties of political theory", en J. Elster y A. Hylland (eds.), Foundations of Social Choice Theory, Cambridge, Cambridge University Press.

(1995), "Forces and Mechanisms in the Constitution-Making Process", Duke Law Review, n" 45, pp. 364-396.

FEARON, J. D. (1998), "Deliberation as Discussion”, en J. Elster (ed.), Deliberative Democracy, Cambridge, Cambridge University Press.

GAMBETTA, D. (1998), “Claro!: An Essay on Discursive Machismo”, en J. Elster (ed.), Deliberative Democracy, Cambridge, Cambridge University Press.

HARDIN, R. (1993), “The Street-level Epistemology of Trust”, Politics and Society, n 21 (4), pp. 505-529.

(1995), One for All. The Logic of Group Conflict, Princeton, Princeton University Press.

HERREROS, F. (2000), "Social capital, Associations and Civic Republicanism”, en M. Saward (ed.), Democratic Innovation: Deliberation, Association and Representation, Londres, Routledge.

HOBBES, T. (1992), Leviatán, Madrid, Alianza Editorial.

INGLEHART, R. (1990), Culture Shift in advanced industrial society, Princeton, Princeton University Press.

JACKMAN, R. W. y R.A. Miller (1996), “A renaissance of political culture?”, American Journal of Political Science, $\mathrm{n}^{\circ} 40$ (3), pp. 632-659.

KREPS, D. (1990), "Corporate culture and economic theory”, en J. Alt y K. Shepsle (eds.), Perspectives on Positive Political Economy, Cambridge, Cambridge University Press. 
RIS

LEVI, M. (1996), "Social and Unsocial Capital: A Review Essay of Robert Putnam's Making Democracy Work", Politics and Society, n 24 (1), pp. 45-55.

MANSBRIDGE, J. J. (1990), "On the Relation of Altruism and Self-Interest", en J.J. Mansbridge (eds.), Beyond Self-Interest, Chicago y Londres, The University of Chicago Press.

MAQUIAVELO, N. (1992), El Príncipe, Madrid, Alianza Editorial.

NORTH, D.C. (1990), Institutions, Institutional Change and Economic Performance, Cambridge, Cambridge University Press.

PITKIN, H. F. (1984), Fortune is a Woman. Gender and Politics in the Thought of Niccolo Machiavelli, Berkeley y Los Angeles, University of California Press.

PUTNAM, R. D. (1993), Making Democracy Work. Civic Traditions in Modern Italy, Princeton, Princeton University Press.

(2000), Bowling Alone. The Collapse and Revival of American Community, New York, Simon and Schuster.

RAWLS, J. (1995), "The Domain of the Political and Overlapping Consensus", en J. E. Roemer, J. Hampton y D. Copp (eds.), The Idea of Democracy, Cambridge, Cambridge University Press.

ROUSSEAU, J.J. (1990), Discurso sobre el origen y los fundamentos de la desigualdad entre los hombres, Madrid, Tecnos.

STOLLE, D. (2000), "Social Capital -A New Research Agenda? Toward an Attitudinal Approach", ponencia presentada en la mesa de "Capital social y asociaciones voluntarias", Copenhague, ECPR Joint Sessions.

STOLLE, D. y T. R. ROCHON (1998), "Are all asociations alike? Member diversity, associational type and the creation of social capital", American Behavioral Scientists, $\mathrm{n}^{\circ} 42$ (1), pp. 47-65.

SUNSTEIN, C. R. (1988), "Beyond the Republican Revival”, Yale Law Journal, n 97, 15391590.

SZTOMPKA, P. (1999), Trust. A Sociological Theory, Cambridge, Cambridge University Press.

TAYLOR, M. (1988), "Revolutionary Collective Action", en M. Taylor (ed), Rationality and Revolution, Cambridge, Cambridge University Press.

TVERSKY,A. y D. KAHNEMAN (1986), "Beliefs in the Law of Small Numbers", en D. Kahneman, P. Slovic y A. Tversky (eds.), Judgement under Uncertainty: Heuristics and Biases, Cambridge, Cambridge University Press.

USLANER, E. M. (1999), “Democracy and Social Capital”, en M. E. Warren (ed.), Democracy and Trust, Cambridge, Cambridge University Press.

WEINGAST, B. R. (1997), "Political Foundations of Democracy and the Rule of Law", American Political Science Review, n⿳0 91, pp. 245-263. 
WHITELEY, P. F. (1999), "The Origins of Social Capital", en J. W. V. Deth, M. Maraffi, K. Newton y P. F. Whiteley (eds.), Social Capital and European Democracy, Londres, Routledge.

WILLIAMSON, O. (1985), The Economic Institutions of Capitalism, New York, The Free Press.

WOOD, G. S. (1987), The Creation of the American Republic. 1776-1787, Williamsburg, Virginia, The University of North Carolina Press.

YAMAGISHI, T. (2001), "Trust as a Form of Social Intelligence”, en K. Cook (ed), Trust in Society, New York, Russell Sage Foundation. 